Keywords: tumour-associated antigen; autoantibodies; DNA-topoisomerase I; early diagnosis of carcinoma; TOPO48; anti-TOPO48 autoantibodies

\title{
A protein fragment derived from DNA-topoisomerase I as a novel tumour-associated antigen for the detection of early stage carcinoma
}

\author{
Shang-mian Yie ${ }^{\star}, 1,2,3$, Shang-rong $\mathrm{Ye}^{1,2}$, Xiao-li Ma ${ }^{2}, \mathrm{Ke} \mathrm{Xie}^{3}$, Jian-bo Zhang ${ }^{4}$, Mei Cao ${ }^{4}, \mathrm{Xu} \mathrm{He}^{4}$, Zhen-bo Hu${ }^{5}$, \\ Cheng-lu Yang ${ }^{2}$, Jia Zhang ${ }^{2}$ and Jie Zeng ${ }^{2}$ \\ ${ }^{1}$ Chengdu Cancer Bioengineering Research Institute, 37 Twelve Bridge Road, Chengdu 610075, Sichuan, PR China; ${ }^{2}$ Chengdu \\ Geimmica Scientific Ltd, Chengdu, Sichuan, PR China; ${ }^{3}$ Department of Oncology, Sichuan Academy of Medical Sciences, \\ Chengdu, Sichuan, PR China; ${ }^{4}$ Core Laboratory, Sichuan Academy of Medical Sciences, Chengdu, Sichuan, PR China and \\ ${ }^{5}$ Laboratory of Stem Cells and Regeneration Medicine, The Affiliated Hospital of Weifang Medical University, Weifang, Shandong, \\ PR China
}

Background: The production of autoantibodies against tumour-associated antigens (TAAs) is believed to reflect greater immunologic reactivity in cancer patients and enhanced immune surveillance for cancer cells. Over the past few decades, a number of different TAAs and their corresponding autoantibodies have been investigated. However, positive frequency of autoantibody detection in cancer patients has been relatively low. Here we describe a novel TAA that was a fragment derived from human DNAtopoiomerase I and an autoantibody against the novel TAA with relatively high positive frequency in the sera of early-stage nonsmall-cell lung cancer (NSCLC), gastric cancer (GC), colorectal cancer (CRC) and oesophageal squamous cell carcinoma (ESCC).

Methods: Serologic enzyme-linked immunosorbent assay (ELISA) and western blot were used to discover a novel TAA with a molecular weight of $48 \mathrm{kDa}$ separated by ion exchange chromatography. Autoantibody ELISA, immnohistochemistry and immunofluorescent staining, recombinant protein cloning/expression and western blot were used to identify the novel TAA. The association of the autoantibody against the novel TAA with early-stage carcinoma was explored by screening 203 stage I/II patients and 170 stage III/IV patients with NSCLC, GC, CRC or ESCC.

Results: We identified the novel TAA as a fragment derived from human DNA-topoiomerase I (TOP1). We found that the novel TAA induced specific autoantibodies with a high prevalence that ranged from 58 to $72 \%$ in some of the most common types of cancer. We observed that the immune response against the novel TAA was associated with early stage ESCC, GC, CRC and NSCLC.

Conclusions: The findings in this study suggest that the autoantibody against the novel TAA may be a potential biomarker for use in the early detection and diagnosis of cancer.

Cancer is a major public health issue around the world (Jemal et al, 2011). According to recent surveys, cancer is now the leading cause of death in China where it is estimated that 4292000 new cancer cases and 2814000 cancer deaths have occurred in 2015 (Chen et al, 2016).
Owing to the lack of perceivable symptoms in the early stages of tumorigenesis, most cancer patients are diagnosed in the late/ distant stages resulting in low survival rates (Bradley et al, 2001). However, many solid tumours are potentially curable if diagnosed

*Correspondence: Professor S-m Yie; E-mail: yieshangmian@yahoo.ca

Received 12 July 2016; revised 7 September 2016; accepted 8 October 2016; published online 22 November 2016

(c) 2016 Cancer Research UK. All rights reserved 0007 - 0920/16 
at an earlier stage when the tumour can be completely removed by surgical means. Unfortunately, efforts at early detection methods, such as imaging technologies or serum soluble protein tumour markers, have failed to provide any significant reduction in cancer mortality rates. Thus, there is a need to develop improved methods that can aid in the early detection of cancer.

One approach to early detection is the discovery of biomarkers that can both identify patients with cancer and assist in appropriate staging. Circulating autoantibodies to tumour-associated antigens (TAAs) represent one class of potentially useful biomarkers. This is so because increased levels of the autoantibodies are found in very early cancer stages (Zayakin et al, 2013), while low incidences of the autoantibodies occur in healthy individuals (Reuschenbach et al, 2009).

Over the past few decades, a number of different TAAs have been investigated (Reuschenbach et al, 2009; Zaenker and, Ziman, 2013). However, in these studies, positive frequency of autoantibody detection in cancer patients has been relatively low, with a frequency range from 0 to $69 \%$ and a $14 \%$ median (Reuschenbach et al, 2009).

It has been reported that a TAA with a molecular weight of $10 \mathrm{kDa}$ called malignin was isolated by using an ion exchange chromatography with stepwise gradient elution (Bogoch, 1977). The detection of antimalignin autoantibodies was reported to be 97\% sensitive and 95\% specific for certain malignancies (Bogoch et al, 1982; Bogoch et al, 1988; Abrams et al, 1994).

In the present study, we report on a novel TAA that was unexpectedly discovered when we tried to isolate malignin by using a similar chromatography method. We identified the novel TAA as a protein fragment derived from human DNA-topoiomerase 1 (TOP1). We found that the novel TAA induced autoantibodies with a high prevalence that ranged from 58 to $72 \%$ in some of the most common types of cancer. Moreover, the autoantibody against the novel TAA was shown to be associated with early stage oesophageal squamous cell carcinoma (ESCC), gastric cancer (GC), colorectal cancer (CRC) and non-small-cell lung cancer (NSCLC).

\section{MATERIALS AND METHODS}

Ion exchange chromatography. To prepare cell/tissue lysates, cancer cell lines were propagated in RPMI medium 1640 supplemented with $10 \% \mathrm{FBS}, 50 \mathrm{U} \mathrm{ml}^{-1}$ penicillin and $50 \mu \mathrm{g} \mathrm{ml}^{-1}$ streptomycin. The cells were then washed with ice cold phosphatebuffered saline (PBS) and lysed on ice in $50 \mathrm{~mm}$ Tris $(\mathrm{pH}=7.5$ at $\left.25^{\circ} \mathrm{C}\right), 150 \mathrm{~mm} \mathrm{NaCl}, 1 \%$ Triton X-100 and proteolytic inhibitors, including aprotinin $\left(1 \mu \mathrm{g} \mathrm{ml}^{-1}\right)$, leupeptin $\left(1 \mu \mathrm{g} \mathrm{ml}^{-1}\right)$, pepstatin $\left(1 \mu \mathrm{g} \mathrm{ml}^{-1}\right)$ and phenylmethylsulphonyl fluoride $\left(50 \mu \mathrm{g} \mathrm{ml}^{-1}\right)$. For cancer tissue lysates, the tissues were washed several times with $0.9 \%$ sodium chloride to remove traces of blood and then minced. This was followed by homogenisation in the same lysate buffer as above with a homogeniser (IKA Ultra-Turrax T25, Stockholm, Sweden). The lysates were centrifuged at 10000 r.p.m. for $20 \mathrm{~min}$ twice. Supernatant was collected through a syringe fitted with a $0.45-\mu \mathrm{m}$ filter.

A Q-Sephadex anion-exchange fast flow resin (Sigma, St Louis, MO, USA) was used to separate proteins in the lysates. The lysates were first adsorbed on the resin, washed with $0.01 \mathrm{M} \mathrm{PBS}$ at $\mathrm{pH} 7.0$ and then eluted by stepwise PBS with a gradient of phosphate concentrations and $\mathrm{pH}$ ( $\mathrm{pH}$ ranged from 4.7 to 4.1 and phosphate concentrations ranged from 0.01 to $1 \mathrm{M}$ ) as well as a $1 \mathrm{~m}$ phosphoric acid solution $(\mathrm{pH}=1)$. Fractions of the lysates were collected every $3 \mathrm{ml}$, and protein concentrations in each of the fractions were determined by a Bio-Rad protein assay-dye reagent kit (Bio-Rad Laboratories, Hercules, CA, USA).
Antibody capture enzyme-linked immunosorbent assay (antibody capture ELISA). Purified proteins from the ion exchange chromatography or purified recombinant proteins from an $\mathrm{Ni}$ sepharose fast flow affinity chromatography (GE, Fairfield, CT, USA) were coated on 96-well microtiter plates (Thermo Labs Systems, Franklin, MA, USA) in $50 \mu \mathrm{l}$ per well in bicarbonate/ carbonate coating buffer $(100 \mathrm{~mm}, \mathrm{pH}=9.6)$. These were left overnight at $4{ }^{\circ} \mathrm{C}$ and un-reacted sites were blocked with a $5 \%$ skim milk solution for $4 \mathrm{~h}$ at room temperature. Serum samples from cancer patients, healthy donors acting as controls, patients with benign tumours or patients tested positive for different autoantibodies associated with autoimmune diseases were diluted at $1: 100$ in PBS. Additionally, a specific rabbit polyclone antibody against the novel TAA (see Supplementary Materials S1, Supplementary Figure S1a) or anti-SCL-70 autoantibodies (Meridian Life Science Inc., Memphis, TN, USA) were diluted to different concentrations in PBS. The diluted serum samples or antibodies were then added to the wells ( $50 \mu$ l per well) and incubated for $1 \mathrm{~h}$ at room temperature. Next, four washes with PBS containing $0.1 \%$ Tween-20 were performed followed by incubation with $50 \mu \mathrm{l}$ horseradish peroxidase (HRP)-conjugated rabbit anti-human IgG or HRP-conjugated goat anti-rabbit antibody (Sigma) diluted at 1:5000 or $1: 1000$ in PBS for another $1 \mathrm{~h}$ at room temperature. Unbound antibodies were removed after four washes. Colour was developed by the addition of $100 \mu$ l tetramethylbenzidine solution (Sigma) for $10 \mathrm{~min}$ at room temperature and stopped by the addition of $100 \mu \mathrm{l}$ of $1 \mathrm{M} \mathrm{HCl}$. The absorbance was read on a micotiter reader (Bio-TEK Instruments, Winooki, VT, USA) at 450/630 nm.

Western blot analysis. To obtain serum immunoblots of the cancer patients, the fractions of the lysates from the ion exchange chromatography were first separated by electrophoresis on $12 \%$ SDS-polyacrylamine gel (SDS-PAGE), and then electrctroblotted to polyvinylidene fluoride (PVDF) membranes (Invitrogen, Carlsbad, CA, USA) at $100 \mathrm{~mA}$ for $1 \mathrm{~h}$ at room temperature. The PVDF membranes were blocked with 5\% milk in PBS, pH 7.4 and incubated with a 1:20 serum pool from the cancer patients overnight at $4{ }^{\circ} \mathrm{C}$. Afterward, the PVDF membranes were washed and incubated with a $1: 1000$ dilution of HRP-conjugated rabbit anti-human IgG for $1 \mathrm{~h}$ at room temperature. After a further four times washing, binding of the primary antibody was revealed by using a $3.3^{\prime}$ diaminobenzidine (DAB) substrate kit (Thermo Labs Systems) in accordance with the manufacturer's instructions.

To obtain the immunoblot with the rabbit polyclonal antibody against the novel TAA or the anti-SCL-70 autoantibodies (Meridian Life Science), the recombinant human TOP1 fragments were separated on the SDS-PAGE and electroblotted to the PVDF membranes as described above. Western blot analyses were performed with 1:100 diluted anti-novel TAA or anti-SCL-70 autoantibodies and HRP conjugated goat anti-rabbit IgG (Sigma) or HRP-conjugated rabbit anti-human IgG. Antigen-antibody complexes were visualised using the DAB substrate kit as described above.

Immnohistochemistry and immunofluorescent staining. For immunohistochemistry, $4-\mu$ m-thick sections of the paraffinembedded tissue blocks were cut and mounted on poly-lysinecoated slides. The slides were dewaxed in xylene and rehydrated through a graded series of ethanol. After deparaffinisation, antigen retrieval treatment was performed for $5 \mathrm{~min}$ at $120^{\circ} \mathrm{C}$ (autoclave) in a $10 \mathrm{nmoll}^{-1}$ sodium citrate buffer ( $\mathrm{pH}$ 6.0). Endogenous peroxidase activity was blocked by using a $0.03 \%$ hydrogen peroxide solution containing sodium azide for $30 \mathrm{~min}$ at room temperature. Next, the rabbit polyclonal antibody against the novel TAA diluted at $1: 100$ was incubated overnight at $4{ }^{\circ} \mathrm{C}$. This was followed by a thorough washing in a $0.01 \mathrm{M}$ PBS solution. Subsequently, binding sites of the primary antibody were visualised by using a Dako EnVison Kit (Dako, Glostrup, Denmark) in 
accordance with the manufacturer's instructions. Finally, sections were faintly counterstained with haematoxylin and mounted with glycerol gelatin. Negative controls were obtained by replacing the primary antibody with normal rabbit serum, or by pre-incubating the rabbit antibody with $30 \mu \mathrm{g} \mathrm{ml}^{-1}$ of the chromatographically purified novel TAA before tissue staining.

For immunofluorescent staining, the cells were washed with PBS and fixed with 4\% ice-cold paraformaldehyde for $10 \mathrm{~min}$. Following three washes with PBS, the fixed cells were incubated for $1 \mathrm{~h}$ at room temperature with the rabbit polyclonal antibody against the novel TAA in an antibody dilution solution (Dako). After incubation with the primary antibodies and washing three times with PBS, the cells were incubated with FITC-conjugated goat anti-rabbit antibodies (Sc2012; Santa Cruz Biotechnology Inc., Dallas, TX, USA) diluted at $1: 1000$ in PBS-3\% (w/v) BSA for $1 \mathrm{~h}$ at room temperature. Following another three times washing with PBS, the nuclei were stained with DAPI, and visualised under a fluorescence microscope (Leica M205 FA; Leica Microsystems, Wetzlar, Germany). Negative controls were prepared by the omission of the primary antibodies with no fluorescent signals being detected.

Cloning, expression and purification of recombinant TOP1 protein fragments. Four fragments of human TOP1 mRNA were amplified by using RT-PCR (see Supplementary Material S2 and Supplementary Material Table S1)) and directionally cloned into PQE 30 UA expression vectors (Qiagen, Hilden, Germany). Recombinant proteins were expressed in Escherichia coli M15 cells (Qiagen) and purified by using the Ni sepharose fast flow affinity chromatography (see Supplement Materials S2 in detail and Supplementary Figure S2a).

Study population and samples. A total of 764 healthy donors, 628 cancer patients with various types of carcinoma and 63 patients with benign tumours were involved in the study to demonstrate the novel TAA. The subject demographics are shown in Supplementary Materials S3 and Supplementary Table S1.

A total of 375 cancer patients with ESCC $(N=107)$, GC $(N=54)$, CRC $(N=87)$ and NSCLC $(N=127)$, diagnosed and treated at three hospitals located in Chengdu, Sichuan, PR China, were involved in the study of the association of the autoantibodies with early-stage carcinoma. The patients comprised 273 males and 102 females with a median age of 58 years old (range $26-86$ years). Of the cancer patients, 55 ESCC, 47 CRC, 29 GC and 71 NSCLC patients were found to be in the early stages (I/II) of cancer, while 52 ESCC, 40 CRC, 25 GC and 56 NSCLC patients suffered from late stages (III/IV) of cancer. None of the cancer patients had any preoperative chemotherapy or radiotherapy before testing. Three hundred and seventy-five age- and gender-matched healthy volunteers, who were confirmed to be cancer-free through clinical imaging and laboratory examinations, were used as controls in the study (see Supplementary Materials S3 and Supplementary Table S2).

Following informed consent, blood samples were collected into collection tubes (see Supplementary Materials S4). After centrifugation for $10 \mathrm{~min}$ at $250 \mathrm{~g}$ and $4{ }^{\circ} \mathrm{C}$ to obtain serum, the serum samples were stored at $-80{ }^{\circ} \mathrm{C}$ until analysis. The study was approved and monitored by the ethics committee at each of the three hospitals (see Supplementary Materials S4).

Statistical analysis. Statistical analysis was performed by using the SPSS software package (14th Edition, Abacus Concepts, Berkeley, CA, USA). We compared the differences in the levels of the autoantibodies between the healthy controls and cancer patients, as well as between the healthy controls and early- and late-stages of cancer by using a standard nonparametric Mann-Whitney $U$ - test. We used the Pearson $\chi^{2}$ test to determine statistical significance between early- and late-stage diseases. We assessed the feasibility of using the autoantibodies as a diagnostic tool for differentiating between malignant cases and healthy controls by using a receiveroperating characteristic (ROC) curve analysis. $P<0.05$ was considered to be significant.
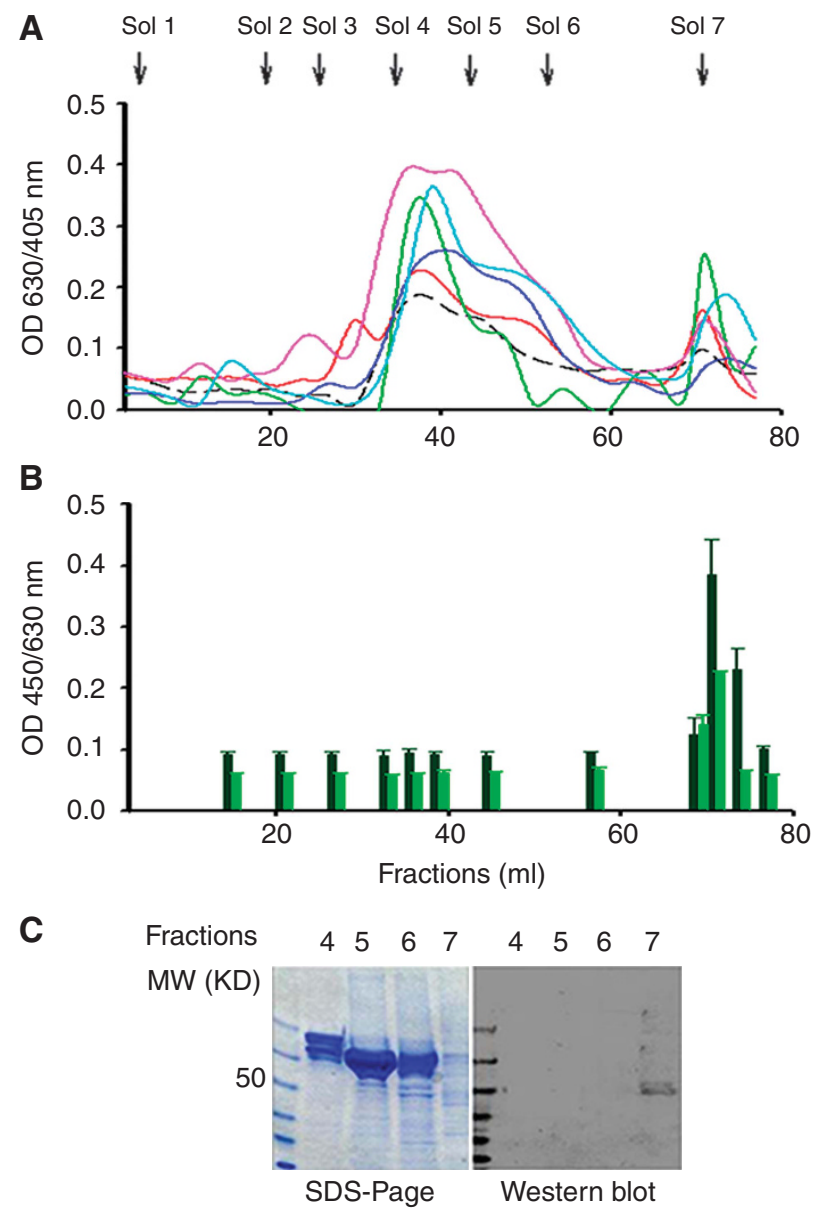

Figure 1. Discovery of a novel TAA by ion exchange chromatography, and serologic ELISA and western blot. (A) Protein profiles of Q-Shepharose ion exchange chromatography stepwise eluted with gradient concentrations of phosphate. Sol $1=$ elution solution 1 (0.005 м PBS, pH =7), Sol 2 = elution solution 2 ( $\left.0.028 \mathrm{M} \mathrm{Na}_{2} \mathrm{HPO}_{3}\right)$, Sol 3 =elution solution $3\left(0.05 \mathrm{M} \mathrm{Na} 2 \mathrm{HPO}_{3}\right)$, Sol $4=$ elution solution 4 $\left(0.175 \mathrm{M} \mathrm{Na}_{2} \mathrm{HPO}_{3}\right)$, Sol $5=$ elution solution $5\left(0.3 \mathrm{M} \mathrm{Na} \mathrm{HPO}_{3}\right)$, Sol $6=$ elution solution $6\left(1.0 \mathrm{M} \mathrm{Na}{ }_{2} \mathrm{HPO}_{3}\right)$ and Sol $7=$ elution solution 7 $\left(1.0 \mathrm{M} \mathrm{H}_{2} \mathrm{PO}_{3}\right.$ ). The profile lines represent cell/tissue lysates of breast cancer cell line MCF-7 (-----), oesophageal squamous cell cancer $(-)$, gastric cancer $(-)$, colorectal cancer $(-)$, non-small cell lung cancer (-) and breast cancer (-), respectively. The $y$-axis represents protein concentrations as measured by a BioRed protein kit at OD $630 / 405 \mathrm{~nm}$, while the $x$-axis represents elution fractions collected every $3 \mathrm{ml}$. (B) The eluted fractions were screened by using an antibody capture ELISA with serum pools obtained from cancer patients or healthy donors. In the eluted fractions of 23-25 (69-74 ml) after adding elution solution 7, there was significantly higher immunoreactivity in the serums of cancer patients ( $\square$ ) when compared with the serums of healthy donors ( $)$. (C) The left image shows chromatographically isolated proteins from fractions 12 (lane 2), 15 (lane 3), 18 (lane 4) and 24 (lane 5) that were subjected to 12\% SDSPAGE separation and stained with Coomassie brilliant blue. The right image shows the same SDS-PAGE that was electrctroblotted to PVDF membranes and immune blotted with 1:20 cancer patient serums. Molecular mass markers $(\mathrm{kDa})$ are shown in lane 1. 


\section{RESULTS}

Discovery of novel TAA. Initially, we used the ion exchange chromatography approach with the stepwise gradient elution, as reported byBogoch (1977), to try to isolate malignin from three human breast cancer cell line MCF-7, MDA-MB-435 and MDAMB-231 and various human carcinoma tissues. All elution profiles were similar among the different lysates of cell lines and carcinoma tissues (Figure 1A). We then used the antibody capture ELISA coated with proteins from the different lysate fractions to detect the immune reactivity in serum pools from cancer patients or healthy donors. The cancer serum pool contained 30 neat serum samples from various common types of carcinoma at different stages, including NSCLC, GC, CRC, ESCC and breast carcinoma (BC). What we found was a significantly higher immune reactivity in the serum pool of the cancer patients with the coated proteins in the fraction of the seventh stepwise elution in all of the tested cell lines and carcinoma tissues when compared with the serum pool of the health donors (Figure 1B).

At first, we thought we had isolated malignin from the cancer cells/tissues by using the ion exchange chromatography. However, unexpectedly, we found that the molecular size of the isolated protein was approximately $48 \mathrm{kDa}$ instead of the reported $10 \mathrm{kDa}$ when we tried to verify the protein by using western blot with the serum pool of the cancer patients (Figure 1C). As such, we hypothesised that the isolated protein was not malignin but another type of TAA.

Identification of novel TAA. To test and verify our hypothesis, we first needed to determine whether the isolated protein was a tumour antigen per se, even though it reacted with antibodies in the serum pool of the cancer patients. To this end, we used the antibody capture ELISA coated with the chromatographically purified protein to measure individual serum samples including 764 healthy donors, 628 cancer patients with various types of carcinoma and 63 patients with benign tumours (see Supplement Materials S3 and Supplementary Table S1). None of the subjects suffered from any autoimmune disorders, which was confirmed by clinical and laboratory examinations. We found that the immune reactivity in the samples from the cancer patients with various types of carcinomas was significantly higher when compared with those from the healthy donors and patients with benign tumours (all $P<0.01$; Figure 2A). Further, when the mean reactive value (OD $450 / 640 \mathrm{~nm}$ ) of the health donors plus 2 s.d. was taken as a cutoff point, the positive prevalence of immune reactivity ranged from $58 \%$ to $72 \%$ depending on the type of carcinoma (Figure $2 \mathrm{~B}$ ). Accordingly, this showed that the isolated protein could be a novel TAA that can induce a high frequency of immune response in various types of carcinoma.

It has been reported that many identified TAAs were derived mostly from cytoplasmic proteins followed by nucleoproteins, membrane-bound proteins and extracellular proteins (Reuschenbach et al, 2009). As such, we examined the cellular location of the novel TAA by using immnohistochemistry and immunofluorescent staining with the rabbit polyclonal antibody. Development of the antibody and its specificity as well as identicalness with the antigen-specific autoantibodies in the cancer patient was demonstrated by using western blot and immune-precipitation/western blot (see Supplement Materials S1 and Supplementary Figure $\mathrm{S} 1 \mathrm{~b}$ and $\mathrm{c})$. The results showed that the novel TAA was predominately expressed in the nucleus of all the types of cancer cells we examined (Figure 3). This suggested that in addition to having a different molecular size than malignin, the novel TAA was a nuclear antigen, and thus different from malignin that was stained in cytoplasmic and membranes of a variety of human cancer cells by anti-malignin antibody with immunofluorescence (Bogoch et al, 1982, 1988).

Moreover, to demonstrate that the novel TAA was not associated with autoimmune diseases, we screened a number of autoantibody-positive serums from patients with autoimmune diseases by using the antibody capture ELISA coated with the novel TAA. As expected, the novel TAA did not react with the autoantibodies in the autoimmune disease serum samples except in the serum samples belonging to the cancer patients (Figure 4A). However, unexpectedly again, we found that the novel TAA reacted with anti-extractable nuclear antibodies (ENAs).

Since ENAs are made up of a group of autoantibodies against autoantigens including ribonucleoproteins (RNP) and non-histone proteins, such as Sm, Jo1, SS-A, SS-B and SCL-70 (Damoiseaux and Tervaert, 2006), we further tested which specific anti-ENA antibody reacted to the novel TAA by using commercially available purified human ENAs (Meridian Life Science Inc.). Figure 4B shows that the novel TAA reacted with anti-SCL-70 autoantibodies but not others. Furthermore, we found that high concentrations of the anti-SCL-70 autoantibodies would compete with the binding of the rabbit antibody to the novel TAA (Figure 4C).

Anti-SCL-70 autoantibodies are linked to scleroderma, which is a chronic autoimmune rheumatic disease (Harris and Rosen, 2003; Gabrielli et al, 2009). The antigenic target of the anti-SCL-70 autoantibodies is DNA-topoisomerase I or TOP1 (Guldner et al, 1986). In early reports, the anti-SCL-70 autoantibodies were found
A

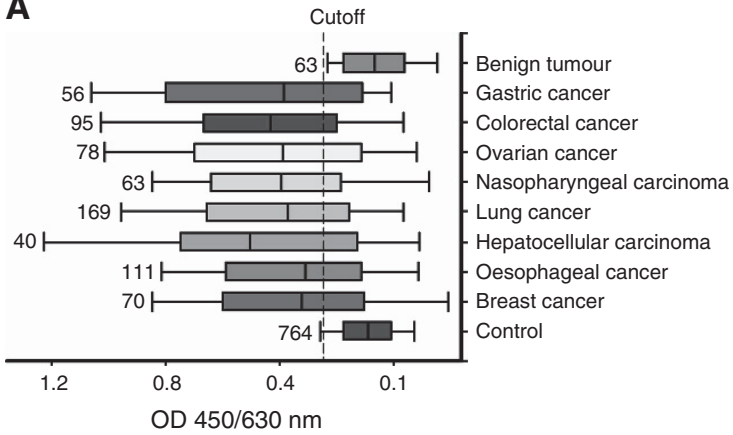

B

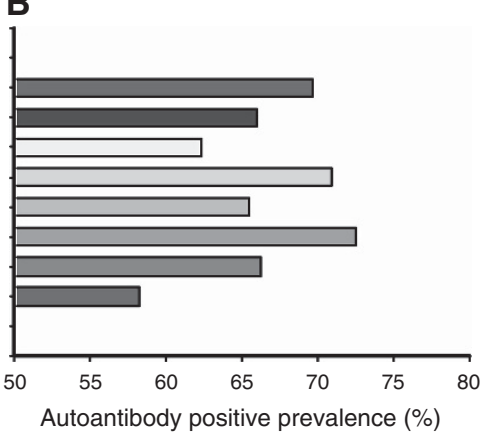

Figure 2. Determination of the novel TAA by a large clinical cohort study. (A) The chromatographically isolated protein (fraction 24) was coated on microtiter plates. Immune reactivity of the isolated protein in individual serum samples from healthy donors and cancer patients or patients with benign tumours were measured by the antibody capture ELISA. Mean reactive value (OD 450/630 nm) of the healthy donors plus 2 s.d. was defined as a cutoff value (vertical short dash line). The numbers on the left side of each bar indicate how many patients of each type of cancer or how many healthy donors were involved in the study. (B) The positive prevalence (\%) of immune reaction in each type of cancer when the cutoff value was used. 
A
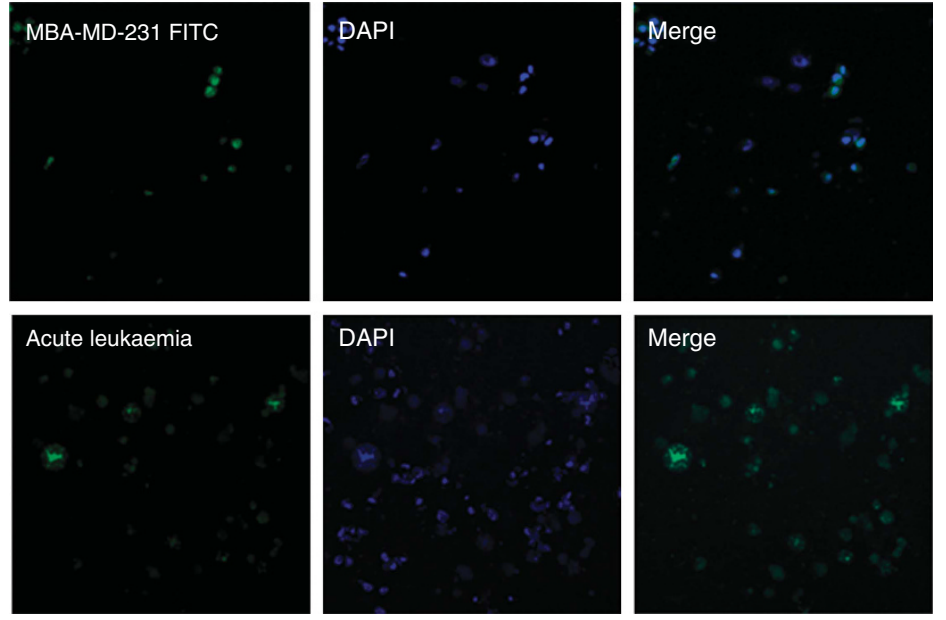

B
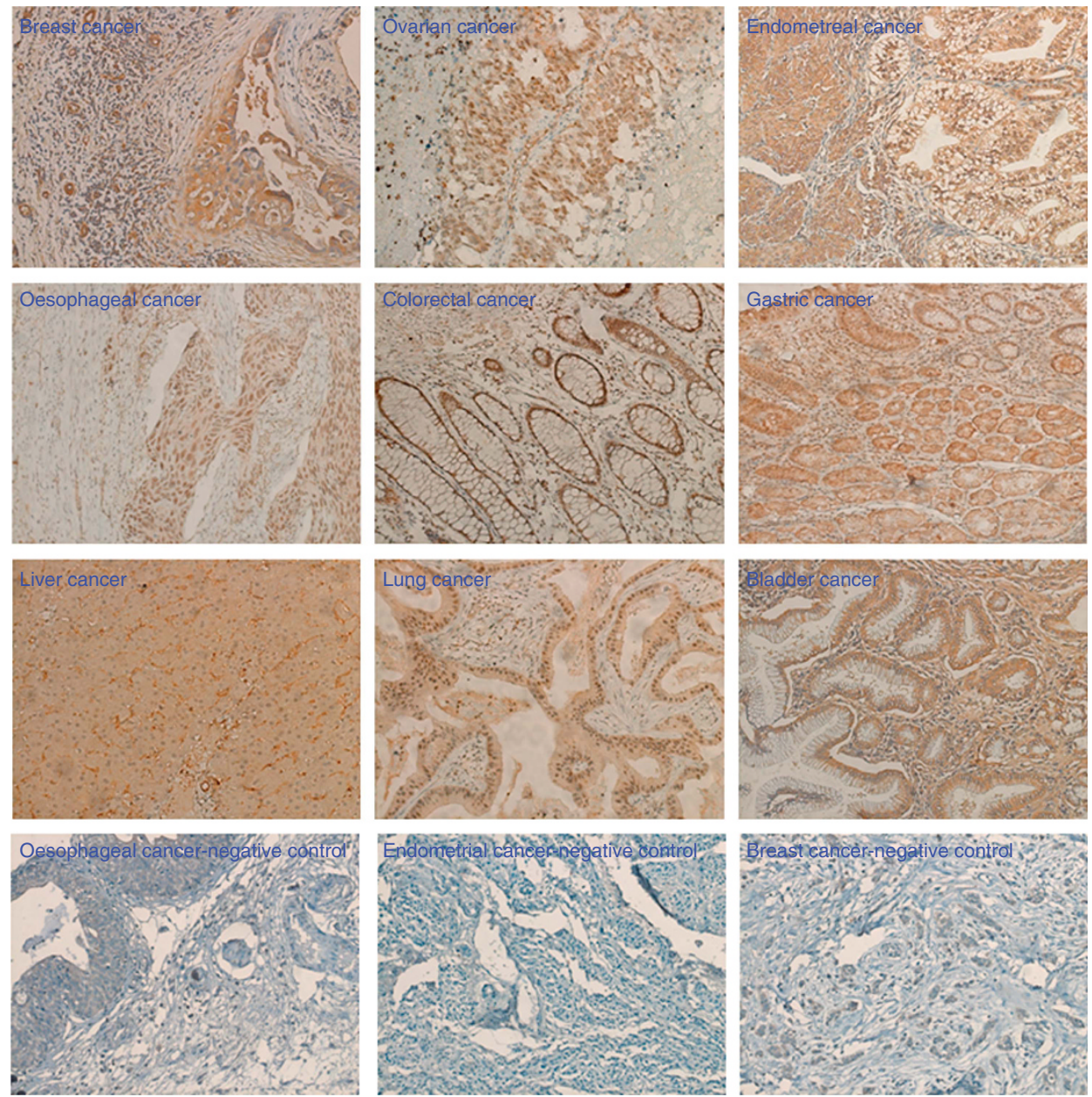

Figure 3. Detection of expression and cellular location of the novel TAA by fluorescence immunostaining and immunohistochemistry. (A) The expression and cellular location of the novel TAA in the MBA-MD-231 breast cancer cell line and acute leukaemia were examined by using fluorescence immunostaining with rabbit polyclonal antibody against the novel TAA. Original magnification at $\times 100$. (B) The expression and cellar location of the novel TAA were detected by immunohistochemistry in various types of human carcinoma. Original magnification at $\times 200$.

to specifically recognise a $70 \mathrm{kDa}$ protein fragment of TOP1, but later reports showed that it can also across-react with the 66-95 kDa protein fragments of TOP1(Guldner et al, 1986). Thus, our results raised two important questions: (1) whether the novel TAA is a fragment of TOP1 and (2) whether the autoantibodies against the novel TAA are distinct from the anti-SCL-70 autoantibodies.

To answer the first question, we cloned and expressed four fragments of TOP1 according to its amino acid sequence (see the Supplement Materials S2 and Supplementary Figure S2b). We then 
A

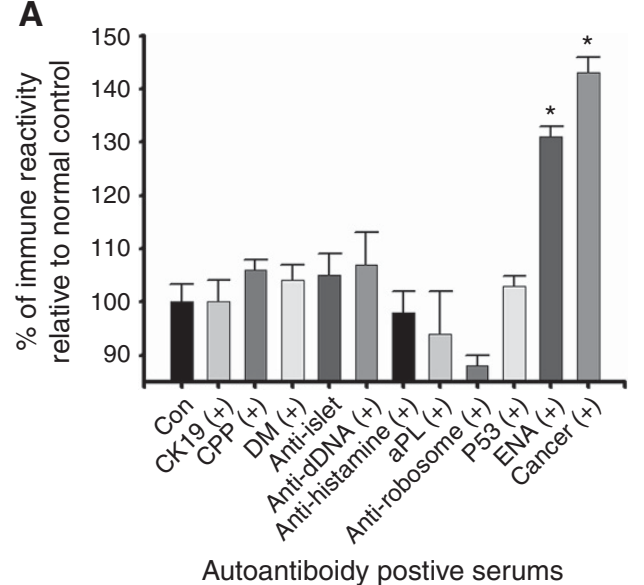

B
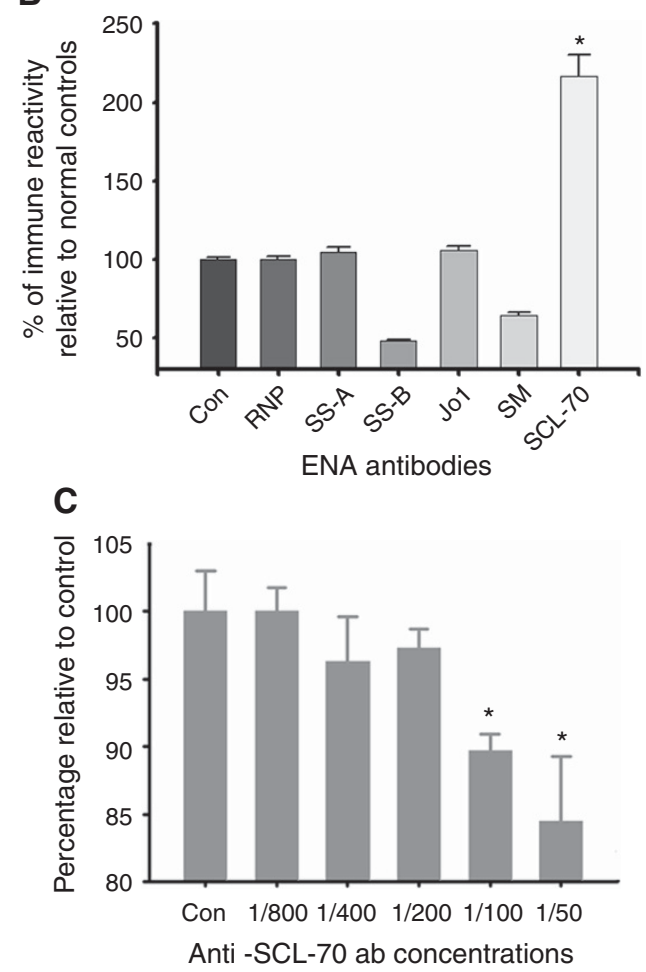

Figure 4. Comparison of immunoreactivity of the novel TAA with several autoantibodies. (A) Comparison of the immune activity against the purified novel TAA in serum samples from healthy controls (Con), cancer patients and patients with positive autoantibodies against CK19, cyclic citrullinated peptide (CCP), dermatomyositis (DM), islet cells, double-stranded DNA (dDNA), histamine, phosphatidylethanolamine $(\mathrm{PE})$, ribosome, p53 and extractable nucleus (EN). The results represent the mean + s.e. for each group ( ${ }^{\star} P<0.001$ as compared with controls). (B) Comparison of the immune activity of the autoantibodies against the novel TAA with the autoantibodies against RNP, SS-A, SS-B, Jo1, SM and SCL-70. The results represent the mean +s.e. for each group $\left({ }^{\star} P<0.001\right.$ as compared with healthy controls). (C) Comparison of the autoantibodies against the novel TAA with the anti-SCL-70 autoantibodies and the rabbit polyclonal antibody. The novel TAA $\left(2 \mu \mathrm{g} \mathrm{ml}^{-1}, 50 \mu \mathrm{l}\right.$ per well) was coated onto microtiter plates. 1:1000 dilution of the rabbit polyclonal antibody was incubated together with various concentrations of the anti-SCL-70 autoantibodies. The results represent the mean + s.e. for each group $\left({ }^{*} P<0.001\right.$ as compared with controls without the anti-SCL-70 autoantibodies). used western blot analysis and the antibody capture ELISA to identify which fragments reacted with the rabbit antibody against the novel TAA, and compared the results with anti-SCL-70 autoantibodies. As shown in Figure 5A and B, the anti-SCL-70 autoantibodies recognised fragments of the TOP1 amino acid sequence 1-209, 192-415 and $410-558$, while the rabbit antibody bonded to fragments of the TOP 1 amino acid sequence $410-558$ and 559-765 (Figure 5C and D). On the one hand, the results showed that the novel TAA is a TOP1 fragment in the amino acid sequence 410-765. On the another hand, the results explained why the novel TAA reacted with the anti-SCL-70 autoantibodies as the rabbit antibody and the anti-SCL-70 autoantibodies both recognised fragments of the TOP1 amino acid sequence $410-558$.

To answer the second question, we used the ELISA coated with the four recombinant TOP1 fragments (labelled fragments 1 to 4 ) to measure 20 serum samples from cancer patients who reacted positively to the novel TAA, 20 serum samples from scleroderma patients who were anti-SCL-70 antibody positive and 20 serum samples from healthy donors. As shown in Figure 5E, no significant differences were seen in the immune reaction to the fragments 2 and 3 when the scleroderma patients' samples were compared with the cancer patients' samples. However, significant differences were observed in the immune reaction to the fragments 1 and 4, where the immune reaction of the scleroderma patients' samples was higher than the cancer patients' samples in the case of fragment $1 \quad(P=0.001)$, while the immune reaction of the scleroderma patients' samples was lower than the cancer patients' samples in the case of fragment $4(P=0.017)$. Figure $5 \mathrm{E}$ also shows that the immune reaction of the scleroderma patients' samples was significantly higher than that of the healthy donors with respect to the fragments 1,2 and 3, but not to the fragment 4 . By contrast, the immune reaction of the cancer patients' samples was significantly higher than that of healthy donors to the fragments 2,3 and 4, but not to the fragment 1 . This not only demonstrated that the autoantibodies against the novel TAA in cancer patients were different from the anti-SCL-70 autoantibodies, but also suggested that the novel TAA amino acid sequence may include parts of fragment 2. Together with the western blot analyses (see Figure 1C and Supplementary Figure S1b and $c$ and Supplement Materials original Wbs), we reasoned that the novel TAA amino acid sequence may be around $329-765$ of the TOP1 protein with a molecular weight of approximately $48 \mathrm{kDa}$.

Finally, to test whether the anti-novel TAA autoantibody can equally recognise the TOP1 recombinant fragment and the chromatographically purified TOP1 fragment, we coated the microtiter plates with the TOP1 recombinant fragment with the amino acid sequence 329-765 and the chromatographically purified protein, respectively. We simultaneously measured and compared 180 serum samples from cancer patients and healthy donors. As shown in Figure 5F, the measurement results were found to be almost the same (regression coefficient $r=0.9281$ ). However, the affinity of the rabbit antibody against the novel TAA to the TOP1 recombinant fragment was significantly less than to the chromatographically purified protein (Figure 5G). This indicated that the novel TAA might have undergone a structural modification after being cleaved from the TOP1 molecule.

From the above results, we concluded that the novel TAA isolated from the ion exchange chromatography was a TOP1 fragment in the amino acid sequence 329-765. The autoantibodies induced by the novel TAA in cancer patients were distinct from the anti-SCL-70 autoantibodies, although the anti-SCL-70 autoantibodies could partially crossreact to the novel TAA. Hence, we named the novel TAA as 'TOPO48' and the autoantibodies against the novel TAA as 'anti-TOPO48 autoantibodies'.

Association of anti-TOPO48 autoantibody with early-stage carcinoma. To explore the clinic significance of the detection of anti-TOPO48 autoantibodies, we developed a special antibody 
A

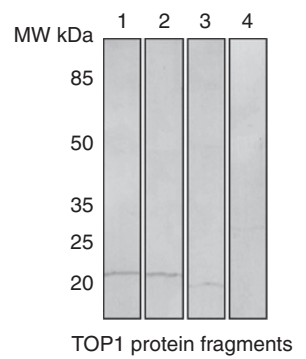

B

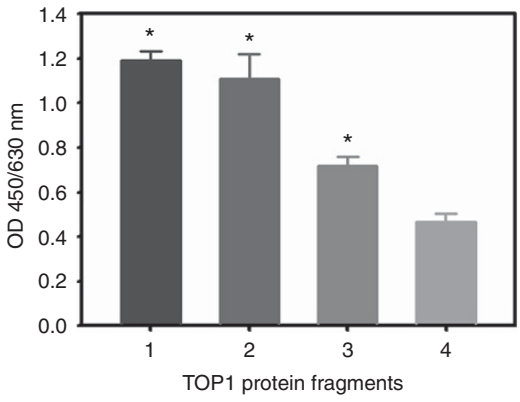

C

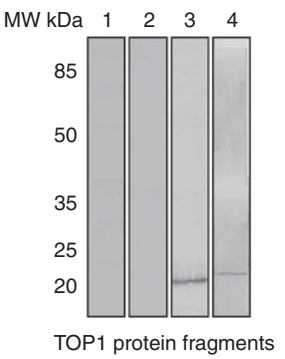

D

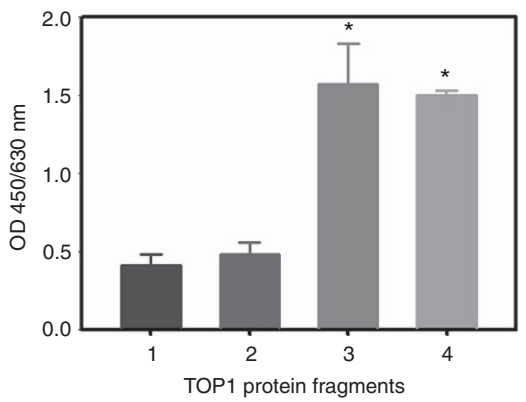

E

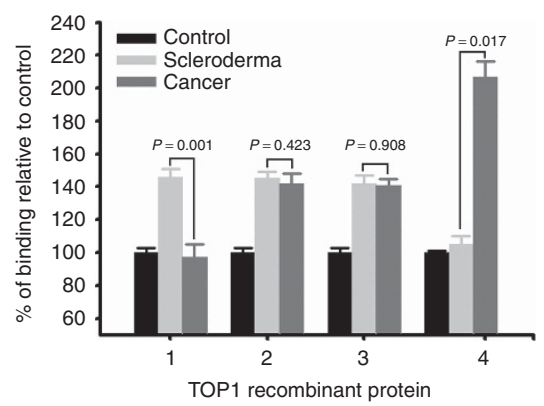

$\mathbf{F}$

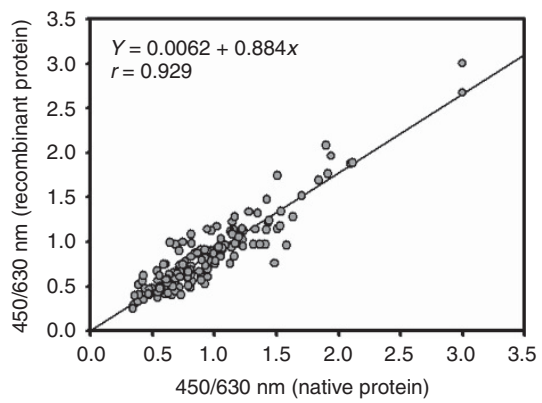

G

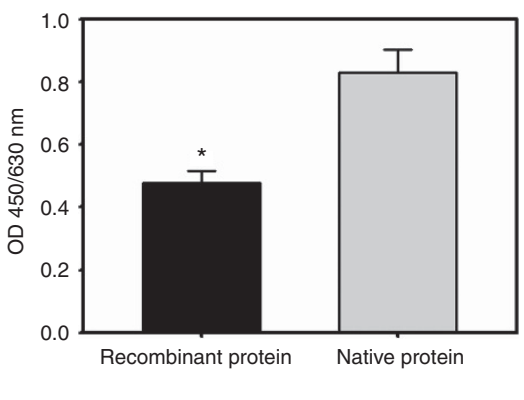

Figure 5. Identification of the novel TAA as a fragment of human DNA-topoisomerase I. (A) Western blot analysis of the anti-SCL-70 autoantibody reactions with four recombinant fragments of TOP1. (B) ELISA analysis of the anti-SCL-70 autoantibody reactions with four recombinant fragments of TOP1. The results represent the mean + s.e. for each group ( $\left.{ }^{\star} P<0.001\right)$. (C) Western blot analysis of the rabbit polyclonal antibody reactions with four recombinant fragments of TOP1. (D) ELISA analysis of the rabbit polyclonal antibody reactions with four recombinant fragments of TOP1. The results represent the mean + s.e. for each group $\left({ }^{*} P<0.001\right)$. (E) Comparison of serum immune reaction to recombinant TOP1 fragments among cancer patients, scleroderma patients and healthy donors. The immune reactions in the serums of the cancer patients and scleroderma patients are presented as percentages of mean + s.e. relative to the serums of the healthy controls for each fragment. The $P$-values indicate the comparisons of the immune reactions in serums between the cancer patients and the scleroderma patients to the each recombinant fragment. (F) One hundred and eighty serum (180) samples were collected from cancer patients, healthy donors and other non-cancer patients. Serum autoantibody against the recombinant TOP1 amino acid sequence 329-765 fragment and the chromatographically purified novel TAA were determined simultaneously, with the correlation being analysed using a linear regression technique. A strong correlation was observed $(y=0.00062+0.844 X, r=0.929, P<0.0001)$. (G) Comparison of antibody affinity of the rabbit anti-novel TAA antibody to the recombinant TOP1 amino acid sequence 329-765 fragment and the chromatographically purified novel TAA. The results represent the mean + s.e. for each group $\left({ }^{*} P<0.001\right)$.

capture ELISA coated with the chromatographically purified protein (see Supplement Materials S3).

To confirm the specificity of the special antibody capture ELISA, we pre-absorbed serums from the cancer patients with chromatographically purified protein before testing them with the ELISA. A discernable decrease of the signals in the ELISA after preabsorption was found (Supplementary Figure S3a). We also used immunoblot to further verify the specificity, and demonstrated that serums from the cancer patients reacted with the TOPO48 protein, while serums from health donors had no such reactivity (Supplementary Figure S3b).

We defined serum immune activity as $\mathrm{U} \mathrm{ml}^{-1}$ when compared with the autoantibody calibrators. The calibrators were purified from the serum pool of cancer patients with an affinity chromatographic column bounded with the TOPO48 recombinant protein (Supplementary Figure S3c).

By using the special antibody capture ELISA, we found that the anti-TOPO48 autoantibody levels in NSCLC, GC, CRC and ESCC were significantly higher than those in the control patients $(P=0.0001$; Figure $6 \mathrm{~A})$. The median value of the anti-TOPO48 autoantibody levels in the healthy controls were $1.11 \mathrm{U} \mathrm{ml}^{-1}$ (range: $0.33-1.42$ ). The median values of the anti-TOPO48 autoantibody levels in the early stages (I and II) of ESCC, GC, CRC and NSCLC were $1.52 \mathrm{Uml}^{-1}$ (range: 0.78-2.62),
$1.68 \mathrm{U} \mathrm{ml}^{-1}$ (range: $0.40-2.66$ ), $1.57 \mathrm{U} \mathrm{ml}^{-1}$ (range: 0.52-2.74) and $1.64 \mathrm{U} \mathrm{ml}^{-1}$ (range: $0.63-3.54$ ), respectively. The median values of the anti-TOPO48 autoantibody levels in the advanced stages (III and IV) of ESCC, GC, CRC and NSCLC were $1.31 \mathrm{U} \mathrm{ml}^{-1}$ (range: $0.55-2.55$ ), $1.43 \mathrm{U} \mathrm{ml}^{-1}$ (range: $0.50-2.64$ ), $1.53 \mathrm{U} \mathrm{ml}^{-1}$ (range: $0.52-2.81$ ) and $1.45 \mathrm{U} \mathrm{ml}^{-1}$ (range: $0.40-$ 2.46), respectively. The anti-TOPO 48 autoantibody levels in the early stages were significantly higher when compared with those in the advanced stages for NSCLC and ESCC $(P=0.022$ and 0.001 , respectively), but not for GC and $\mathrm{CRC}$ (Figure 6A).

To be considered anti-TOPO-48 autoantibody positive, the autoantibody concentrations in serums had to be greater than a cutoff point, which was defined as the maximum value of the control serum immune activity $\left(1.42 \mathrm{U} \mathrm{ml}^{-1}\right)$. Based on this criterion, as shown in Figure 6B, we found that the percentage of positive anti-TOPO48 autoantibody serums in the early stages of cancer was significantly higher than in the late stages: $61.8 \%$ vs $38.2 \%$ (ESSC), $69.0 \%$ vs $31.0 \%$ (GC), $72.3 \%$ vs $27.7 \%$ (CRC) and $67.6 \%$ vs $32.4 \%$ (NSCLC).

Finally, AUC values of the ROC analysis of autoantibody levels for early stage cancer patients versus healthy controls were 0.875 $(95 \% \mathrm{CI}=0.796-0.955), 0.847$ (95\% CI $=0.727-0.967), 0.832(95 \%$ $\mathrm{CI}=0.742-0.923)$ and $0.990(95 \% \mathrm{CI}=0.980-0.999)$ for $\mathrm{ESCC}$, GC, CRC and NSCLC, respectively (Figure 6C-F). 
A

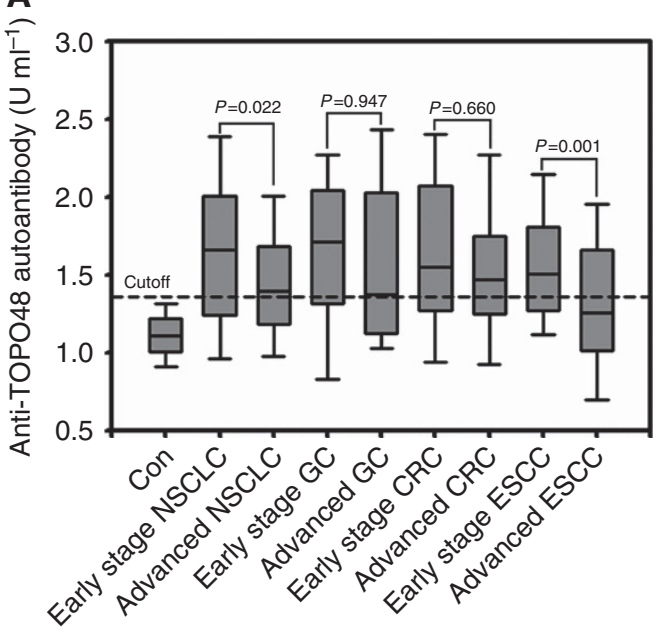

B

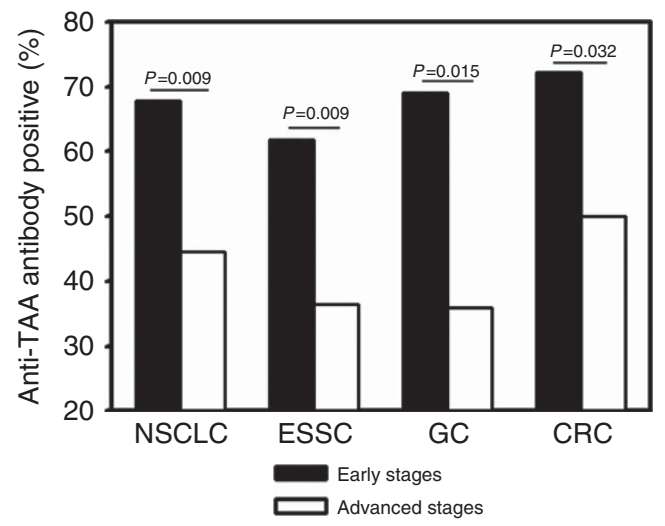

C

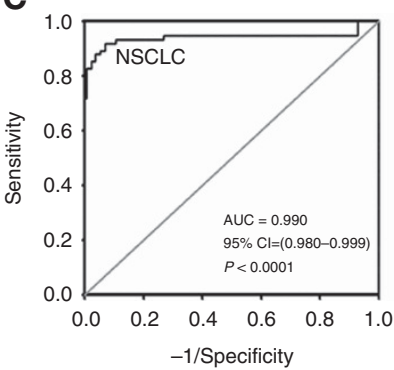

D

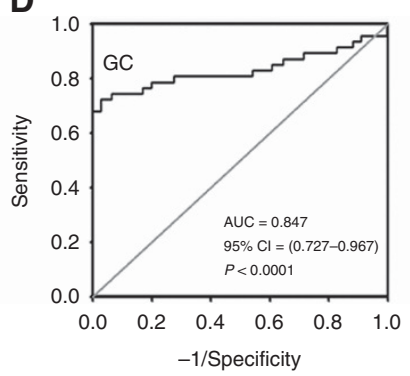

E

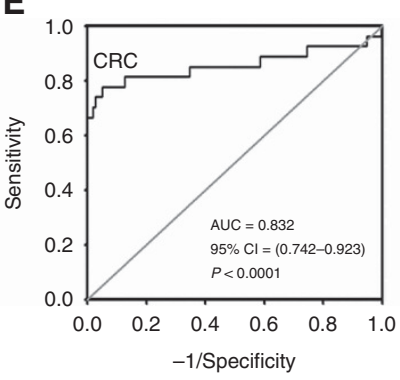

F

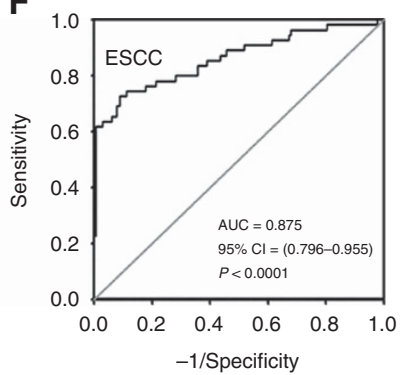

Figure 6. Association of the autoantibodies against the novel TAA with early-stage carcinomas. (A) Serum levels of the autoantibodies against TOPO48 in healthy controls and patients with early and late stages of ESSC, GC, CRC and NSCLC were assessed by using the ELISA. Median levels of serum anti-TOPO48 autoantibody ranges are illustrated by box plot. The maximum value of the immune activity in the healthy controls was defined as a cutoff value (horizontal short dash line). The $P$-values indicate the comparisons of the immune reactions in serums between the early and late stages of each type of carcinoma. (B) Percentages of serum samples with positive TOPO48 autoantibody in early stages (I/II) and late stages (III/IV) of ESCC, GC, CRC and NSCLC. The cutoff point was defined as $1.42 \mathrm{U} \mathrm{ml}^{-1}$. The $P$-values indicate the comparisons of the percentage of serum samples with positive TOPO48 autoantibody between the early and late stages of each type of carcinoma. (C-F) ROC analysis for the autoantibody levels for healthy controls versus early stage NSCLC, GC, CRC and ESCC, respectively.

\section{DISCUSSION}

In the present study, we discovered a novel TAA, which we named TOPO48. We determined that the novel TAA had a molecular weight of $48 \mathrm{kDa}$, and identified it as a fragment of TOP1 in the amino acid sequence $329-765$. DNA-topoiomerase I is a nuclear enzyme that plays an essential role in alleviating topological stresses that arise during DNA replication and transcription. DNAtopoiomerase I per se is an autoantigen that induces anti-SCL-70 autoantibodies in scleroderma (Jimenez and Derk, 2004) as well as other autoimmune diseases (Ho and Reveille, 2003; Mahler et al, 2010). However, anti-SCL-70 autoantibodies are not associated with the occurrence of cancer even if TOP1 is overexpressed in a wide range of cancers (Ikeguchi et al, 2011), or if there is a temporal clustering of scleroderma and/or other cancers (Shah et al, 2010; Joseph et al, 2014). Our results demonstrated that the autoantibodies against the novel TAA in cancer patients are not anti-SCL-70 autoantibodies. Although the anti-SCL-70 autoantibodies do have a partial crossreaction with the 1-229 amino acid sequence in the novel TAA, which corresponds to the 329-558 amino acid sequence in TOP1.

It is important to understand how the novel TAA emerged in cancer patients. It is unlikely that the $48 \mathrm{kDa}$ fragment we found is an artificial product that was produced under a very low $\mathrm{pH}$ environment during the chromatographic separation process. For example, a single band of the $48 \mathrm{kDa}$ fragment could always be detected in western blot with whatever rabbit antibody or autoantibodies in the cancer patient serums without undergoing the chromatographic separation process.

Studies have established that the disturbance of self-tolerance is a major mechanism for autoimmune diseases (Kamradt and Mitchison, 2001). The immune responses against self-antigens that occur in cancer patients are most likely different from primary autoimmune diseases (Reuschenbach et al, 2009). In addition to the loss of self-tolerance, the immune responses in cancer patients may be initiated by alterations in the tumour itself, which result in increased immunogenicity of the self-antigens (Fonseca and Dranoff, 2008). These alterations include coding DNA mutations that can lead to new epitopes in expressed proteins, posttranslational modifications with immunological relevance, and altered tissue specific expression patterns or levels that can lead to the exposure of antigens that are normally expressed solely in immuno-privileged sites (Houghton, 1994; Renkvist et al, 2001; Jäger et al, 2003; Reuschenbach et al, 2009; Zaenker and Ziman, 2013). Moreover, some studies have suggested that the cleavage of known nuclear and cytoplasmic proteins during apoptosis and/or the accumulation due to insufficient clearance of secondary necrotic cells may play a role in the immune responses of cancer patients (Edinger and Thompson, 2004; Vogelstein and Kinzler, 2004).

TOP1 releases DNA topological stress during DNA transcription and replication through the formation of DNA-TOP1 complexes, transient cleavages and subsequent relegation of one strand of the DNA helix (Champoux, 2001). The formation of the 
complexes is potentially dangerous to cells leading to genomic instability and oncogenesis (Larsen and Gobert, 1999). Thus, the level and stability of these complexes have to be strictly regulated in non-transformed cells by maintaining relatively constant enzyme levels, limiting the stability of the cleavable complexes through interactions with other proteins such as p53, and degrading the TOP1 protein by proteasomes (Larsen and Gobert, 1999).

As discussed above, TOP1 is overexpressed in a wide range of cancers (Ikeguchi et al, 2011). This overexpression may alter the proteasome-mediated degradation of enzymes in neoplastic cells since the enzymes are regulated differently than in normal or cancer cells (Larsen and Gobert, 1999). In light of this and together with our findings in the present study, it is possible that the novel TAA is one of the TOP1 proteasome degradation products whose protein structures have been altered through unknown mechanisms after proteolysis. The alteration may trigger immune responses and the production of specific autoantibodies when cryptic determinants are revealed during tumour development and progression. However, further studies are needed to verify this hypothesis.

Nevertheless, in the present study, we revealed that the autoantibodies against the novel TAA are associated with early stages in ESSC, GC, CRC and NSCLC. The association with early stages and higher autoantibody positive rate suggest a potential clinical application for using the autoantibodies as an early diagnostic tool for ESSC, GC, CRC and NSCLC. The levels of the autoantibodies against TOPO48 were higher in the early stages than those in the advanced stages for ESCC and NSCLC, but not for GC and CRC. However, the percentage of anti-TOP1 autoantibody positive cases was higher in early stages than in advanced stages for all the four types of carcinoma. These results suggested that there were more early stage cancer patients having detectable humoral immune response to TOPO48 than advanced stage patients regardless of the tumour loads. Moreover, it remains uncertain whether the association with early stages also exists in other types of cancer. Therefore, more clinical studies with appropriate designs are necessary to determine the connection that the novel TAA has with other types of cancer. Finally, the association between the occurrence of the novel TAA and autoantibody production in early stages of cancer needs to be evaluated in prospective clinical studies.

It has been proposed that antibody-TAA binding may represent the end stage of the mechanism to initiate the destruction of cancer cells containing corresponding antigens by labelling the antigens for faster macrophage recognition and phagocytosis (Zaenker et al, 2016). Direct binding of antibodies to the antigens can also block receptors associated with tumour cell proliferation and survival (Zaenker et al, 2016). Autoantibodies can drive antigen uptake via dendritic cell $\mathrm{Fc}$ gamma receptors, lead to antigen crosspresentation and vigorous $\mathrm{CD} 4+$ and $\mathrm{CD} 8+\mathrm{T}$ cell responses, and complement dependent cytotoxicity and natural killer cellmediated antibody-dependent cellular cytotoxicity (Carter, 2001). Thus, it should be interesting to explore in future studies whether the autoantibodies against the novel TAA are also involved in various host defense mechanisms that initiate the destruction of cancer cells, limit tumour growth and suppress metastasis.

Moreover, TOP1 is the sole cellular target for chemotherapeutic drugs of the camptothecin (CPT) family that stabilises TOP1DNA complexes and increases DNA strand breaks and unstable RNA transcripts as well as incomplete DNA replications (Torchilin et al, 2001; Koster et al, 2007). DNA-topoiomerase I inhibitor drugs can be effective in some types of cancer (Pommier et al, 2010; Xu and Her, 2015). Accordingly, it is possible that the discovery of the novel TAA may lead to the development of new immune therapeutic agents that can improve patient survival, and at the same time reduce side effects caused by chemotherapeutic drugs.

\section{ACKNOWLEDGEMENTS}

This study was supported by grants from the Research and Development Program of the Chengdu Technology Bureau (No. KJXM20030826).

\section{CONFLICT OF INTEREST}

The authors declare no conflict of interest.

\section{AUTHOR CONTRIBUTIONS}

S-mY and S-rY conceived and designed the experiments. S-mY, $\mathrm{X}-\mathrm{lM}, \mathrm{C}-\mathrm{IY}$ and JZ performed the experiments. S-mY analysed the data. J-bZ, MC, XH, Z-bH and JZ contributed to providing the reagents/materials/analysis tools. KX contributed to collecting clinical data of the studied population. S-mY wrote the paper.

\section{REFERENCES}

Abrams MB, Bednarek KT, Bogoch S, Bogoch ES, Dardik HJ, Dowden R, Fox SC, Goins EE, Goodfried G, Herrman RA (1994) Early detection and monitoring of cancer with the anti-malignin antibody test. Cancer Detect Prev 18: 65-78.

Bogoch S (1977) Astrocytin and malignin: two polypeptide fragments (recognins) related to brain tumor. Natl Cancer Inst Monogr 46: 133-137.

Bogoch S, Bogoch ES, Fager CA, Harris JH, Ambrus JL, Lux WE, Ransohoff JA (1982) Determination of anti-malignin antibody and malignin in 1,026 cancer patients and controls: relation of antibody to survival. J Med 13: 49-69.

Bogoch S, Bogoch ES, Iliescu VM (1988) In vitro production of the general transformation antibody related to survival in human cancer patients: antimalignin antibody. Cancer Detect Prev 12: 313-320.

Bradley CJ, Given CW, Roberts C (2001) Disparities in cancer diagnosis and survival. Cancer 91: 178-188.

Carter P (2001) Improving the efficacy of antibody-based cancer therapies. Nat Rev Cancer 1: 118-129.

Champoux JJ (2001) DNA topoisomerases: structure, function, and mechanism. Annu Rev Biochem 70: 369-413.

Chen W, Zheng R, Baade PD, Zhang S, Zeng H, Bray F, Jemal A, Yu XQ, He J (2016) Cancer statistics in China, 2015. CA Cancer J Clin 66: 115-132.

Damoiseaux JG, Tervaert JW (2006) From ANA to ENA: how to proceed? Autoimmun Rev 5: 10-17.

Edinger AL, Thompson CB (2004) Death by design: apoptosis, necrosis and autophagy. Curr Opin Cell Biol 16: 663-669.

Fonseca C, Dranoff G (2008) Capitalizing on the immunogenicity of dying tumor cells. Clin Cancer Res 14: 1603-1608.

Gabrielli A, Avvedimento EV, Krieg T (2009) Scleroderma. N Engl J Med 360 1989-2003.

Guldner HH, Szostecki C, Vosberg HP, Lakomek HJ, Penner E, Bautz FA (1986) Scl 70 autoantibodies from scleroderma patients recognize a 95 $\mathrm{kDa}$ protein identified as DNA topoisomerase I. Chromosoma 94: 132-138.

Harris ML, Rosen A (2003) Autoimmunity in scleroderma: the origin, pathogenetic role, and clinical significance of autoantibodies. Curr Opin Rheumatol 15: 778-784.

Ho KT, Reveille JD (2003) The clinical relevance of autoantibodies in scleroderma. Arthritis Res Ther 5: 80-93.

Houghton AN (1994) Cancer antigens: immune recognition of self and altered self. $J$ ExpMed 180: 1-4.

Ikeguchi M, Arai Y, Maeta Y, Ashida K, Katano K, Wakatsuki T (2011) Topoisomerase I expression in tumors as a biological marker for CPT-11 chemosensitivity in patients with colorectal cancer. Surg Today 41: 1196-1199.

Jäger E, Jäger D, Knuth A (2003) Antigen-specific immunotherapy and cancer vaccines. Int J Cancer 106: 817-820. 
Jemal A, Bray F, Center MM, Ferlay J, Ward E, Forman D (2011) Global cancer statistics. CA Cancer J Clin 61: 69-90.

Jimenez SA, Derk CT (2004) Following the molecular pathways toward an understanding of the pathogenesis of systemic sclerosis. Ann Intern Med 140: $37-50$.

Joseph CG, Darrah E, Shah AA, Skora AD, Casciola-Rosen LA, Wigley FM, Boin F, Fava A, Thoburn C, Kinde I, Jiao Y, Papadopoulos N, Kinzler KW, Vogelstein B, Rosen A (2014) Association of the autoimmune disease scleroderma with an immunologic response to cancer. Science 343(6167): 152-157.

Kamradt T, Mitchison NA (2001) Tolerance and autoimmunity. N Engl J Med 344: 655-664.

Koster DA, Palle K, Bot ES, Bjornsti MA, Dekker NH (2007) Antitumour drugs impede DNA uncoiling by topoisomerase I. Nature 448: 213-217.

Larsen AK, Gobert C. (1999) DNA topoisomerase I in oncology: Dr Jekyll or Mr Hyde? Pathol Oncol Res 5: 171-178.

Mahler M, Silverman ED, Schulte-Pelkum J, Fritzler MJ (2010) Anti-Scl-70 (topo-I) antibodies in SLE: myth or reality? Autoimmun Rev 9: 756-760.

Pommier Y, Leo E, Zhang H, Marchand C. (2010) DNA topoisomerases and their poisoning by anticancer and antibacterial drugs. Chem Biol 17: 421-433.

Renkvist N, Castelli C, Robbins PF, Parmiani G (2001) A listing of human tumor antigens recognized by $\mathrm{T}$ cells. Cancer Immunol Immunother 50: 3-15.

Reuschenbach M, von Knebel Doeberitz M, Wentzensen N. (2009) A systematic review of humoral immune responses against tumor antigens. Cancer Immunol Immunother 58: 1535-1544.
Shah AA, Rosen A, Hummers L, Wigley F, Casciola-Rosen L (2010) Close temporal relationship between onset of cancer and scleroderma in patients with RNA polymerase I/III antibodies. Arthritis Rheum 62: 2787-2795.

Torchilin VP, Lakoubov LZ, Estov Z (2001) Antinuclear autoantibodies as potential antineoplastic agents. Trends Immunol 22: 424-427.

Vogelstein B, Kinzler KW (2004) Cancer genes and the pathways they control. Nat Med 10: 789-799.

Xu Y, Her C (2015) Inhibition of topoisomerase (DNA) I (TOP1): DNA damage repair and anticancer therapy. Biomolecules 5: 1652-1670.

Zaenker P, Ziman MR (2013) Serologic autoantibodies as diagnostic cancer biomarkers-a review. Cancer Epidemiol Biomarkers Prev 22: 2161-2181.

Zayakin P, Ancāns G, Siliņ a K, Meistere I, Kalniņ a Z, Andrejeva D, Endzeliņ š E, Ivanova L, Pismennaja A, Ruskule A, Doniņ a S, Wex T, Malfertheiner P, Leja M, Linē A (2013) Tumor-associated autoantibody signature for the early detection of gastric cancer. Int J Cancer 132: 137-147.

Zaenker P, Gray ES, Ziman MR (2016) Autoantibody production in cancer the humoral immune response toward autologous antigens in cancer patients. Autoimmun Rev 15: 477-483.

This work is published under the standard license to publish agreement. After 12 months the work will become freely available and the license terms will switch to a Creative Commons AttributionNonCommercial-Share Alike 4.0 Unported License.

Supplementary Information accompanies this paper on British Journal of Cancer website (http://www.nature.com/bjc) 